\title{
The study of new iterations procedure for expansion mappings
}

\author{
Zena Hussein Maibed \\ Reyadh.D.Ali \\ Department of Mathematics \\ College of Education for Pure Science \\ College of Education for Pure Science \\ Ibn Al-Haithem \\ University Of Baghdad \\ Mrs_zena.hussein@yahoo.com \\ University of Karbala \\ reyadhdelphi@gmail.com
}

Recived : $27 \backslash 8 \backslash 2018$

Revised : 24\9\2018

Accepted : $2 \backslash 10 \backslash 2018$

Available online : $\quad 21 / 10 / 2018$

DOI: 10.29304/jqcm.2019.11.1.445

\begin{abstract}
:
In this research, we introduce new iteration process for different types of mappings and introduce a concept of expansion mapping, it is independent of non - expansive mapping . Also, we study the convergences for these iterations to common fixed point in real Hilbert spaces.
\end{abstract}

Keywords: asymptotic fixed point, strong convergence, non-expansive mapping, maximal monotone, weak convergence.

Mathematics Subject Classification: 46S40. 


\section{Introduction}

Let $H$ be a real Hilbert space $\emptyset \neq \mathrm{C} \subseteq H$, and $T: C \rightarrow C$ is non - expansive. Mapping. That is, if $\|a-b\| \geq\|f(a)-f(b)\|$ for $\quad$ each $a, b \in$ $C$. Also any multivalued operator $A$ is called monotone if the following condition hold:

$<a_{1}-a_{2}, d_{1}-d_{1}>\geq 0 \quad \forall a_{i} \in \mathrm{D}(\mathrm{A}), d_{i} \in$ $\mathrm{A}\left(z_{i}\right)$. And it is called maximal monotone if for all $(a, h) \in H \times H,\langle a-b ., . h-d\rangle \geq 0$ and for all $(b, d) \in \operatorname{gph}(\mathrm{A})$ then we get, $h \in \mathrm{A}(z)$. The monotone operators has an important role in different branches of mathematics, see.( [1][5]). On other hand, The convergence of the iteration method studied by many researchers see ([6]-[16]). Define the following mapping as follows:

$J_{r_{n}}=\left(I+r_{n} A^{-1}\right)(a)$ this mapping is called resolvet mapping where $\left\langle r_{n}\right\rangle$ be a sequence of positive real numbers. Also, the metric projection $P_{c}(a)$ from $H$ onto $C$ is defined as follows:

For any $a \in H$ there exists a unique element $P_{c}(a) \in C$ satisfies the following:

$\left\|a-P_{c}(a)\right\| \leq\|a-b\|$, for all $b \in C$. That is, for each $a \in X, P_{c}(a)=b$ iff $b \in C$ and $\|a-b\|=$ $\inf \{\|a-c\| ; c \in C\}$.

Now, the following definitions and lemmas are interesting to area of research:

\section{$\operatorname{Lemma(1.1)~[16]~}$}

Let $\left\langle\alpha_{n}\right\rangle$ and $\left\langle\beta_{n}\right\rangle$ are sequences of nonnegative real number such that $\alpha_{n+1} \leq \alpha_{n}+\beta_{n}$, for each. $n \geq 1$. If $\sum_{n=o}^{\infty} \alpha_{n}$ converge, then $\lim _{n \rightarrow \infty} \alpha_{n}$ exists.

\section{Definition(1.2) : [17]}

Let $\Gamma: C \rightarrow C$ be a mapping then every $p \in C$ is called asymptotic fixed point of $\Gamma$ if there exists $\left\langle\alpha_{n}\right\rangle$ is sequence in $C$ such that $\alpha_{n} \rightarrow p$ and $\left\|\alpha_{n}-\Gamma\left(\alpha_{n}\right)\right\| \rightarrow 0$.

\section{Lemma (1.3) : [18]}

Let $C$ be a nonempty convex closed subset of real Hilbert space $H$ and $\Gamma$ is non-expansive multivalued mapping such that $\operatorname{Fix}(\Gamma) \neq \varnothing$.Then $\Gamma$ is demiclosed, i.e., $\alpha_{n} \rightarrow p$ and $\lim _{n \rightarrow \infty} d\left(\alpha_{n}, \Gamma\left(\alpha_{n}\right)\right)=$ 0.Then $p \in \Gamma(p)$.

\section{Lemma(1.4) : [19]}

If $\left\langle\alpha_{n}\right\rangle$ be a sequence in $H$ and $\left\|\alpha_{n+1}-\alpha\right\| \leq$ $\left\|\alpha_{n}-\alpha\right\|$ for all $\alpha \in C$. Then $\left\langle P_{c}\left(\alpha_{n}\right)\right\rangle$ converges strongly to a point in $C$.

Now, we introduce the concept of expansion mapping

\section{Main Results}

In this section, we define a new iterations for sequence of expansion mapping. Also, we study the convergence for these iterations. 


\section{Definition(2.1)}

Any mapping $f$ is called expansion mapping if for each sequence, $\left\langle z_{n}\right\rangle$ in converges to zero then there exists a nonnegative real number $z$ such that

$$
\begin{aligned}
& \left(1-z_{n}\right)\|x-w\|^{2}+z\left\langle x-f_{x}, w-f_{w}\right\rangle^{k+1} \\
\geq & \|f x-f w\|^{2}, \text { for all } k>0 \text { and } x, w \in C
\end{aligned}
$$

The concept of expansion mapping is independent of non - expansive

mapping. As shown by the following examples:

\section{Example (2.2)}

If $f:(0, \infty) \longrightarrow(0, \infty)$ be a mapping such that $f(x)=x$. Then the mapping $f$ is not non-expansive but it is expansion,mapping. Since, for each sequence $\left\langle z_{n}\right\rangle$ in $[0,1]$ converges to zero then there exists $z$ such that,

$$
\begin{aligned}
& z=\frac{4}{\left\langle x-f_{x}, w-f_{w}\right\rangle^{k+1}}\|x-w\|^{2} \text { and satisfy } \\
& \left(1-z_{n}\right)\|x-w\|^{2}+z\left\langle x-f_{x}, w-f_{w}\right\rangle^{k+1}
\end{aligned}
$$

\section{Example (2.3)}

Let $f: H \longrightarrow H$ be a mapping such that $f(x)=x$.

It is clear that the mapping $f$ is not expansion mapping but it is non - expansive.

\section{Theorem (2.4):}

$$
\text { Let } A_{1}, A_{2}, \ldots ., A_{m} \text { are maximal }
$$
monotone multivalued mapping $C$ nonempty convex closed in $H,\left\langle f_{n}\right\rangle$ be a sequence of non-expansive mapping and $\left\langle T_{n}\right\rangle$ is bounded sequence of expansion mapping on $C$. Let $\left\langle a_{n}\right\rangle,\left\langle b_{n}\right\rangle$ are sequences in $(0,1]$ converges to 0 , such that $a_{n}+b_{n}=1$ and $\sum_{i=1}^{m} \gamma_{n, i}=1$. Define the iteration process $\left\langle x_{n}\right\rangle$ as follows:

$$
w_{n}=b_{n} v_{n}+\left(1-b_{n}\right) \sum_{i=1}^{m} \gamma_{n, i} J_{r_{n, i}}^{i} v_{n}
$$

$$
v_{n+1}=a_{n} T_{n} v_{n}+\left(1-a_{n}\right) f_{n} w_{n}
$$

If $\bigcap_{n=1}^{\infty} F i x\left(J_{r_{n, i}}^{i}\right) \cap\left(\cap_{n=1}^{\infty} F i x\left(T_{n}\right)\right) \cap$

$\left(\bigcap_{n=1}^{\infty} F i x\left(f_{n}\right)\right) \neq \varnothing$. Then $\left\langle x_{n}\right\rangle$ has converges weakly to an asymptotic common fixed point of $T_{n}$ for each $n \in N$. Moreover $\left\langle P_{C}\left(v_{n}\right)\right\rangle$ converges strongly to a point in $C$.

\section{Proof :}

Let $\quad p \in \bigcap_{n=1}^{\infty} F i x\left(J_{r_{n, i}}^{i}\right) \cap\left(\bigcap_{n=1}^{\infty} F i x\left(T_{n}\right)\right) \cap$ $\left(\cap_{n=1}^{\infty} \operatorname{Fix}\left(f_{n}\right)\right)$

$$
\begin{gathered}
\left\|w_{n}-p\right\|^{2}=\left\|\left(\sum_{i=1}^{m} \gamma_{n, i} J_{r_{n, i}}^{i} y_{n}\left(v_{n}\right)-p\right)\right\|^{b_{n}\left(v_{n}-p\right)+\left(1-b_{n}\right) .} \|^{2} \\
\leq b_{n}\left\|v_{n}-p\right\|^{2} \\
\quad+\left(1-b_{n}\right) \sum_{i=1}^{m} \gamma_{n, i}\left\|v_{n}-p\right\|^{2} \\
\leq b_{n}\left\|v_{n}-p\right\|+\left(1-b_{n}\right)\left\|v_{n}-p\right\|^{2} \\
=\left\|v_{n}-p\right\|^{2}
\end{gathered}
$$

Now, for any sequence $\left\langle z_{n}\right\rangle$ in $[0,1]$ converges to zero then there exists a nonnegative real number $Z$ such that

$$
\begin{aligned}
& \left\|v_{n+1}-p\right\|^{2}=\left\|T_{n} v_{n}+\left(1-a_{n}\right) f_{n} w_{n}-p\right\|^{2} \\
& \leq a_{n}\left\|T_{n} v_{n}-p\right\|^{2} \\
& +(1 \\
& \left.-a_{n}\right)\left\|f_{n} w_{n}-p\right\|^{2} \\
& \begin{aligned}
\left\|v_{n+1}-p\right\|^{2} \leq a_{n}\left\|T_{n} v_{n}-p\right\|^{2} \\
+
\end{aligned} \\
& \begin{aligned}
\left\|v_{n+1}-p\right\|^{2} \leq & a_{n}\left(1-a_{n}\right)\left\|w_{n}-p\right\|^{2} \\
& +b_{n} z_{n} \|\left(T_{n} p-p T_{n}\right)\left(v_{n}-v_{n}\right. \\
& \left.-\left(T_{n} p-p\right)\right) \| \\
& +b_{n} z\left\langle v_{n}-f_{x}, p-f_{p}\right\rangle^{k} \\
& +\left(1-a_{n}\right)\left\|v_{n}-p\right\|^{2} \\
\leq & a_{n}\left\{\left(1-z_{n}\right)\left\|v_{n}-p\right\|^{2}\right\} \\
& +\left(1-a_{n}\right)\left\|v_{n}-p\right\|^{2}
\end{aligned}
\end{aligned}
$$




$$
\begin{gathered}
\leq a_{n}\left\|v_{n}-p\right\|^{2}+\left(1-a_{n}\right)\left\|v_{n}-p\right\|^{2} \\
=\left\|v_{n}-p\right\|^{2}
\end{gathered}
$$

By lemma (1.1), we get $\lim _{n \rightarrow \infty}\left\|v_{n}-p\right\|$ exists and hence $\left\langle f_{n}\right\rangle$ is also bounded. So by lemma (1.4) we get $\left\langle P_{C}(v)\right\rangle$ converges strongly to the point in $C$.

$$
\begin{aligned}
\left\|v_{n}-T_{n} v_{n}\right\| \leq \| & a_{n-1}\left(b_{n-1} T_{n-1} v_{n-1}\right. \\
& \left.+\left(1-b_{n-1}\right) f_{n-1} w_{n-1}-T_{n} v_{n}\right) \\
& +\left(1-a_{n-1}\right) f_{n-1} v_{n-1}-T_{n} v_{n} \| \\
& \leq a_{n-1} \| b_{n-1} T_{n-1} v_{n-1} \\
& +\left(1-b_{n-1}\right) T_{n} w_{n-1}-T_{n} v_{n} \| \\
& +b_{n-1}\left\|f_{n-1} v_{n-1}-T_{n} v_{n}\right\|
\end{aligned}
$$

Since $\left\langle f_{n}\right\rangle$ and $\left\langle T_{n}\right\rangle$ are also bounded and $\left\langle a_{n}\right\rangle,\left\langle b_{n}\right\rangle$ are sequences in $(0,1]$ converges to zero. As $n \longrightarrow \infty$ we get, $\left\|v_{n}-T_{n} v_{n}\right\| \longrightarrow 0$.

Now, since $\left\langle v_{n}\right\rangle$ is bounded then there exists subsequence $\left\langle v_{n k}\right\rangle$ of $v_{n}$ such that $v_{n k} \rightarrow z$ and $\left\|v_{n}-T_{n} v_{n}\right\| \longrightarrow 0$. Then we get $z$ is an asymptotic common fixed of $T_{n}$, for each $n \in N$. Then the iteration, $\left\langle v_{n}\right\rangle$ has converges weakly to an asymptotic common fixed point of $T_{n}$, for each $n \in N$.

Now, we consider property $\mathcal{P}$ for any sequence as follows:

Let $\left\langle T_{n}\right\rangle$ be a sequence, of mapping we say that $\left\langle T_{n}\right\rangle$ has property $\mathcal{F}$ if $\left\langle T_{n}\right\rangle$ satisfies the condition:

$\left\|T_{n}-z\right\|^{2} \leq\left\|T_{n}\right\|^{2}$,for each $z \in\left(\cap_{n=1}^{\infty} \operatorname{Fix}\left(T_{n}\right)\right)$.

In the following theorem we study the convergence for the iteration process

$$
\begin{gathered}
w_{n}=b_{n}\left[a_{n}^{\prime} v_{n}+\left(1-a_{n}^{\prime}\right) \sum_{i=1}^{m} \gamma_{n, i} J_{r_{n, i}}^{i} v_{n}\right] \\
+\left(1-b_{n}^{\prime}\right) g_{n} v_{n} \\
v_{n+1} \\
=a_{n}^{\prime}\left[a_{n} T_{n} v_{n}+b_{n} f_{n} v_{n}+c_{n} f_{n} g_{n} v_{n}\right] \\
+b_{n} g_{n} w_{n}
\end{gathered}
$$

where $\left\langle a_{n}^{\prime}\right\rangle$ and $\left\langle b_{n}\right\rangle$ are sequences in $[0,1]$ such that $\left\langle a_{n}^{\prime}\right\rangle,\left\langle b_{n}^{\prime}\right\rangle$ converges to zero, $a_{n}^{\prime} \geq \dot{b}_{n}$. Such that $a_{n}^{\prime}+b_{n}^{\prime}=1, a_{n}+b_{n}+c_{n}=1, \sum_{i=1}^{m} \gamma_{n, i}$.

\section{Theorem (2.5) :}

Let $A_{1}, A_{2}, \ldots . ., A_{m}$ are maximal monotone multivalued mapping and $\emptyset \neq C$ convex closed in $X,\left\langle T_{n}\right\rangle$ is bounded,sequences of expansion mappings on $C$ and $\left\langle f_{n}\right\rangle,\left\langle g_{n}\right\rangle$ are sequences of non-expansive mapping on $C$. If the iteration process defined as (2.1) and $\left(F i x\left(J_{r_{n, i}}^{i}\right)\right) \cap\left(\cap_{n=1}^{\infty} F i x\left(T_{n}\right)\right) \cap\left(\cap_{n=1}^{\infty} F i x\left(f_{n}\right)\right) \cap$ $\left(\bigcap_{n=1}^{\infty} F i x\left(g_{n}\right)\right) \neq \varnothing$. Then $\left\langle x_{n}\right\rangle$ has converges weakly to an asymptotic common fixed point of $T_{n}$, for each $n \in N$. Moreover $\left\langle P_{C}\left(x_{n}\right)\right\rangle$ converges strongly to a point in $C$.

\section{Proof :}

Let

$$
\begin{aligned}
p \in\left(\operatorname{Fix}\left(J_{r_{n, i}}^{i}\right)\right) & \cap\left(\bigcap_{n=1}^{\infty} \operatorname{Fix}\left(T_{n}\right)\right) \cap\left(\bigcap_{n=1}^{\infty} \operatorname{Fix}\left(f_{n}\right)\right) \\
& \cap\left(\bigcap_{n=1}^{\infty} \operatorname{Fix}\left(g_{n}\right)\right)
\end{aligned}
$$

$\left\|w_{n}-p\right\|^{2}$

$$
\begin{aligned}
& \leq \| b_{n}\left[\begin{array}{c}
a_{n}^{\prime}\left(v_{n}-p\right)+\left(1-a_{n}^{\prime}\right) \\
\left(\sum_{i=1}^{m} \gamma_{n, i} J_{r_{n, i}}^{i} v_{n}-p\right) \\
\left(1-b_{n}\right) g_{n} v_{n}-p
\end{array} \|^{2}\right. \\
& \leq \overline{b_{n}}\left\|\left(\sum_{i=1}^{a_{n}^{\prime}\left(v_{n}-p\right)+\left(1-a_{n}^{\prime}\right)} \gamma_{n, i} J_{r_{n, i}}^{i} v_{n}-p\right)\right\|^{2} \\
& +\left(1-\overline{b_{n}}\right)\left\|P_{c} g_{n} v_{n}-p\right\|^{2}
\end{aligned}
$$




$$
\begin{aligned}
&\left\|w_{n}-p\right\|^{2} \leq b_{n} {\left[\begin{array}{c}
a_{n}^{\prime}\left\|v_{n}-p\right\|^{2}+\left(1-a_{n}^{\prime}\right) \\
\sum_{i=1}^{m} \gamma_{n, i}\left\|J_{r_{n}}^{i} v_{n}-p\right\|^{2}
\end{array}\right] } \\
&+\left(1-b_{n}\right)\left\|v_{n}-p\right\|^{2} \\
& \leq \dot{b}_{n}\left[a_{n}^{\prime}\left\|v_{n}-p\right\|^{2}\right. \\
&\left.+\left(1-a_{n}^{\prime}\right)\left\|v_{n}-p\right\|^{2}\right] \\
&+\left(1-\dot{b_{n}}\right)\left\|v_{n}-p\right\|^{2} \\
&\left\|w_{n}-p\right\|^{2}=b_{n}\left\|v_{n}-p\right\|^{2}+\left(1-b_{n}\right)\left\|v_{n}-p\right\|^{2} \\
&=\left\|v_{n}-p\right\|^{2}
\end{aligned}
$$

Hence, $\quad\left\|w_{n}-p\right\|^{2} \leq\left\|v_{n}-p\right\|^{2}$

Now, by (2.1) then we have

$$
\begin{aligned}
\left\|v_{n+1}-p\right\|^{2} \leq & a_{n}^{\prime} \| a_{n} T_{n} v_{n}+b_{n} f_{n} v_{n}+c_{n} f_{n} g_{n} v_{n} \\
& -p\left\|^{2}+b_{n}\right\| g_{n} w_{n}-p \|^{2} \\
\| v_{n+1}- & p\left\|^{2} \leq a_{n}^{\prime} a_{n}\right\| T_{n} v_{n}-p \|^{2} \\
& +a_{n}^{\prime} b_{n}\left\|f_{n} v_{n}-p\right\|^{2} \\
& +a_{n}^{\prime} c_{n}\left\|f_{n} g_{n} v_{n}-p\right\|^{2} \\
& -a_{n}^{\prime} a_{n} b_{n}\left\|T_{n} v_{n}-f_{n} v_{n}\right\|^{2} \\
& -a_{n}^{\prime} b_{n} c_{n}\left\|f_{n} v_{n}-f_{n} T_{n} v_{n}\right\|^{2} \\
& -a_{n}^{\prime} c_{n} a_{n}\left\|f_{n} T_{n} v_{n}-T_{n} v_{n}\right\|^{2} \\
& +b_{n}^{\prime}\left\|g_{n} w_{n}-p\right\|^{2} \\
\leq a_{n}^{\prime} a_{n} \| T_{n} v_{n}- & p\left\|^{2}+a_{n}^{\prime} b_{n}\right\| f_{n} v_{n}-p \|^{2} \\
& +a_{n}^{\prime} c_{n}\left\|f_{n} g_{n} v_{n}-p\right\|^{2} \\
& +a_{n}^{\prime} a_{n} b_{n}\left\|T_{n} v_{n}-f_{n} v_{n}\right\| \\
& +a_{n}^{\prime} b_{n} c_{n}\left\|f_{n} v_{n}-f_{n} T_{n} v_{n}\right\|^{2} \\
-a_{n}^{\prime} c_{n} a_{n} \| f_{n} T_{n} v_{n} & -T_{n} v_{n} \|^{2} \\
& -a_{n}^{\prime} a_{n} b_{n}\left\|T_{n} v_{n}-f_{n} v_{n}\right\| \\
& -a_{n}^{\prime} b_{n} c_{n}\left\|f_{n} v_{n}-f_{n} T_{n} v_{n}\right\|^{2} \\
& -a_{n}^{\prime} c_{n} a_{n}\left\|f_{n} T_{n} v_{n}-T_{n} v_{n}\right\|^{2} \\
& +b_{n}^{\prime}\left\|g_{n} w_{n}-p\right\|^{2}
\end{aligned}
$$

For any sequence $\left\langle z_{n}\right\rangle$ in $[0,1]$ converges to zero there exists a nonnegative real number $z$ such that $\left\|v_{n+1}-p\right\|^{2}$

$$
\begin{aligned}
\leq a_{n}^{\prime} a_{n}\left[\left(1-z_{n}\right)\right. & \left\|v_{n}-p\right\|^{2} \\
& +z_{n}\left\|p-T_{n} p\right\| . \|\left(p-T_{n} p\right)\left({ }_{c} v_{n}\right. \\
& \left.-T_{n} v_{n}-\left(p-T_{n} p\right)\right) \| \\
& \left.+z\left(\left\langle p-T_{n} p, v_{n}-T_{n} v_{n}\right\rangle\right)^{k+1}\right] \\
& +a_{n}^{\prime} b_{n}\left\|v_{n}-p\right\|^{2} \\
& +a_{n}^{\prime} c_{n}\left\|v_{n}-p\right\|^{2} \\
+ & b_{n}\left\|w_{n}-p\right\|^{2}
\end{aligned}
$$

Now,

$$
\begin{aligned}
& \left\|v_{n+1}-p\right\|^{2} \leq a_{n}^{\prime} a_{n} \\
& \left\|v_{n}-p\right\|^{2}+a_{n}^{\prime} b_{n}\left\|v_{n}-p\right\|^{2}+a_{n}^{\prime} c_{n}\left\|v_{n}-p\right\|^{2} \\
& +b_{n}\left\|v_{n}-p\right\|^{2} \\
& \left\|v_{n+1}-p\right\|^{2}=a_{n}^{\prime}\left\|v_{n}-p\right\|^{2}+\left(1-a_{n}^{\prime}\right)\left\|v_{n}-p\right\|^{2} \\
& =\left\|v_{n}-p\right\|^{2}
\end{aligned}
$$

By lemma (1.1),

we get $\lim _{n \rightarrow \infty}\left\|v_{n}-p\right\|$ exists. Hence, $\left\langle v_{n}\right\rangle$ is bounded sequence, so that $\left\langle g_{n}\right\rangle$ and $\left\langle f_{n}\right\rangle$ are also bounded sequences.

So, by lemma (1.4) we deduce $\left\langle P_{C}\left(x_{n}\right)\right\rangle$ converges strongly to the point in $C$.

$$
\begin{aligned}
\left\|v_{n}-T_{n} v_{n}\right\|=\| & a_{n-1}^{\prime}\left[a_{n-1} T_{n-1} v_{n-1}\right. \\
& +b_{n-1} f_{n-1} v_{n-1} \\
& \left.+c_{n-1} f_{n-1} g_{n-1} v_{n-1}\right] \\
& +b_{n-1}^{\prime}\left[a _ { n - 1 } b _ { n - 1 } \left(T_{n-1} v_{n-1}\right.\right. \\
& \left.-f_{n-1} v_{n-1}\right) \\
& +b_{n-1} c_{n-1}\left(f_{n-1} v_{n-1}\right. \\
& \left.-f_{n-1} T_{n-1} v_{n-1}\right) \\
& +c_{n-1} a_{n-1}\left(f_{n-1} T_{n-1} v_{n-1}\right. \\
& \left.\left.-T_{n-1} v_{n-1}\right)+d_{n-1} g_{n-1} w_{n-1}\right] \\
& -T_{n} w_{n} \|
\end{aligned}
$$




$$
\begin{aligned}
&\left\|v_{n}-T_{n} v_{n}\right\| \leq a_{n-1}^{\prime} \| a_{n-1} T_{n-1} v_{n-1} \\
&+b_{n-1} f_{n-1} v_{n-1} \\
&+c_{n-1} f_{n-1} T_{n-1} v_{n-1}-g_{n} w_{n} \| \\
&+b_{n-1}^{\prime} \| a_{n-1} b_{n-1}\left(T_{n-1} v_{n-1}\right. \\
&\left.-f_{n-1} v_{n-1}\right) \\
&+b_{n-1} c_{n-1}\left(f_{n-1} v_{n-1}\right. \\
&\left.-f_{n-1} T_{n-1} v_{n-1}\right) \\
&+c_{n-1} \alpha_{n-1}\left(f_{n-1} T_{n-1} v_{n-1}\right. \\
&\left.-T_{n-1} v_{n-1}\right)+d_{n-1} g_{n-1} w_{n-1} \\
&-T_{n} w_{n} \|
\end{aligned}
$$

Since $\dot{a}_{n}, \dot{b}_{n} \longrightarrow 0 \quad$ and $\left\langle T_{n}\right\rangle,\left\langle f_{n}\right\rangle$ and $\left\langle g_{n}\right\rangle$ are bounded then we get

$$
\left\|v_{n}-T_{n} v_{n}\right\| \longrightarrow 0 \text { as } n \longrightarrow \infty
$$

Now, since $\left\langle v_{n}\right\rangle$ is bounded sequence then there exists subsequence $\left\langle v_{n k}\right\rangle$ of $\left\langle v_{n}\right\rangle$ such that $v_{n k} \rightarrow Z$ and since $\left\|v_{n}-T_{n} v_{n}\right\| \longrightarrow 0$,then we get,

$Z$ is asymptotic common fixed point of $T_{n}$, for all $n \in N$.

Then the iterations $\left\langle v_{n}\right\rangle$ has converges weakly to an asymptotic common fixed point of $T_{n}$, for all $n \in$ $N$.

In the following theorem we give a new iteration process and we study the convergence for this iteration to an asymptotic common fixed point.

\section{Theorem (2.6) :}

If $\left\langle f_{n}\right\rangle$ be a sequence of non-expansive mapping on $\mathrm{C}$ and $\left\langle T_{n}\right\rangle$ be a bounded sequence of expansion mappings on C. Define the iteration $\left\langle v_{n}\right\rangle$ as follows:

$$
\begin{gathered}
w_{n}=a_{n}^{\prime} f_{n} v_{n}+\left(1-a_{n}^{\prime}\right)\left(T_{n} v_{n}\right) \\
v_{n+1}=a_{n} \sum_{i=1}^{m} \gamma_{n, i} J_{r_{n, i}}^{i} v_{n} \\
+\left(1-a_{n}\right) f_{n} w_{n}
\end{gathered}
$$

where $\left\langle a_{n}^{\prime}\right\rangle,\left\langle b_{n}^{\prime}\right\rangle,\left\langle a_{n}\right\rangle,\left\langle b_{n}\right\rangle$ are sequences in $[0,1]$ such that $\left\langle a_{n}\right\rangle,\left\langle b_{n}\right\rangle$ converges to 0 such that. If $\quad\left(\cap_{n=1}^{\infty} F i x\left(J_{r_{n, i}}^{i}\right)\right) \cap\left(\cap_{n=1}^{\infty} F i x\left(T_{n}\right)\right) \cap$ $\left(\cap_{n=1}^{\infty} \operatorname{Fix}\left(f_{n}\right)\right)$

$\neq \varnothing$. Then the iteration process $\left\langle v_{n}\right\rangle$ has converges weakly to an asymptotic common fixed point of $T_{n}$, for all $n \in N . \quad$ Moreover $\quad\left\langle P_{C}\left(v_{n}\right)\right\rangle$ converges, strongly to a point in C.

\section{Proof :}

Let

$$
p \in\left(\operatorname{Fix}\left(P_{c}\right)\right) \cap\left(\cap_{n=1}^{\infty} \operatorname{Fix}\left(T_{n}\right)\right) \cap
$$

$\left(\cap_{n=1}^{\infty} \operatorname{Fix}\left(f_{n}\right)\right)$

Since $w_{n}=a_{n}^{\prime} f_{n} v_{n}+\left(1-a_{n}^{\prime}\right)\left(b_{n} P_{c} T_{n} v_{n}+\right.$ $\left.\left(1-\overline{b_{n}}\right) f_{n} P_{c} T_{n} v_{n}\right)$ then we have,

$$
\begin{aligned}
& \left\|w_{n}-p\right\|^{2} \leq a ́\left\|f_{n} v_{n}-p\right\|^{2} \\
& +\left(1-a_{n}^{\prime}\right)\left[\left\|T_{n} v_{n}-p\right\|^{2}\right] \\
& \left\|w_{n}-p\right\|^{2} \leq a_{n}^{\prime}\left\|v_{n}-p\right\|^{2} \\
& +\left(1-a_{n}^{\prime}\right)\left[\left\|T_{n} v_{n}-p\right\|^{2}\right] \\
& =a_{n}^{\prime}\left\|v_{n}-p\right\|^{2} \\
& +\left(1-a_{n}^{\prime}\right)\left\|T_{n} v_{n}-p\right\|^{2}
\end{aligned}
$$

For any sequence $\left\langle z_{n}\right\rangle$ in $[0,1]$ converges to zero there exists a nonnegative real number $z$ such that

$$
\begin{aligned}
\left\|w_{n}-p\right\|^{2} \leq a_{n}^{\prime} & \left\|v_{n}-p\right\|^{2} \\
& +\left(1-a_{n}^{\prime}\right)\left[\left(1-z_{n}\right)\left\|v_{n}-p\right\|^{2}\right. \\
& +z_{n} \|\left(p-T_{n} p\right)\left(v_{n}-T_{n} v_{n}\right. \\
& \left.-\left(p-T_{n} p\right)\right) \| \\
& \left.+z\left(\left\langle v_{n}-T_{n} v_{n}, p-T_{n} p\right\rangle\right)^{k}\right]
\end{aligned}
$$

$$
\begin{aligned}
\left\|w_{n}-p\right\|^{2} & \leq a ́\left\|v_{n}-p\right\|^{2}+\left(1-a_{n}^{\prime}\right)\left\|v_{n}-p\right\|^{2} \\
& =\left\|v_{n}-p\right\|^{2}
\end{aligned}
$$

Hence, $\left\|w_{n}-p\right\|^{2} \leq\left\|v_{n}-p\right\|^{2}$

$$
\begin{array}{r}
\left\|v_{n+1}-p\right\|^{2} \leq a_{n} \sum_{i=1}^{m} \gamma_{n, i}\left\|J_{r_{n, i}}^{i} v_{n}-p\right\|^{2} \\
+\left(1-a_{n}\right)\left\|f_{n} w_{n}-p\right\|^{2}
\end{array}
$$




$$
\begin{gathered}
\left\|v_{n+1}-p\right\|^{2} \leq a_{n} \sum_{i=1}^{m} \gamma_{n, i}\left\|v_{n}-p\right\|^{2} \\
+\left(1-a_{n}\right)\left\|w_{n}-p\right\|^{2} \\
\left\|v_{n+1}-p\right\|^{2} \leq a_{n}\left\|v_{n}-p\right\|^{2} \\
+\left(1-a_{n}\right)\left\|v_{n}-p\right\|^{2} \\
=\left\|v_{n}-p\right\|^{2}
\end{gathered}
$$

By lemma (1.1), we get $\lim _{n \rightarrow \infty}\left\|v_{n}-p\right\|$ exists

Hence, the iteration $\left\langle x_{n}\right\rangle$ is bounded sequence. So $\left\langle f_{n}\right\rangle$ and $\left\langle g_{n}\right\rangle$ also bounded sequences. And hence, by lemma (1.4) we deduce $\left\langle P_{C}\left(v_{n}\right)\right\rangle$ converges strongly to a point in $C$.

\section{References}

1 . H.H. Bauschke and P.L. Combettes, Convex Analysis and Monotone Operator Theory in Hilbert Spaces, Springer-Verlag, 2011.

2. J.M. Borwein and J.D. Vanderwerff, Convex Functions, Cambridge University Press, 2010.

3. R.S. Burachik and A.N. Iusem, Set-Valued Mappings and Enlargements of Monotone Operators, Springer-Verlag, 2008. 24.

4. S. Simons, From Hahn-Banach to Monotonicity, Springer-Verlag, 2008.

5. C. $Z$ alinescu, Convex Analysis in General Vector Spaces, world Scientific Publishing, 2002.

6. H.K.Xu, a nother control condition in an iterative method for nonexpansive mappings, bull ,austral. Math .soc ,65 (2002),pp:109-113.

7. H.K.Xu,Iterative Algorithm for nonlinear operators ,J. London.Math.Soc. (2002) pp:240256.
8. A.Moudafi, Viscosity approximation method for fixed point problems, Journal of Mathematical Analysis and Applications, 241(2000) pp:46-55.

9. H.K.Xu,Viscosity approximation methods for nonexpansive

$$
\text { mapping }
$$

J.Math.Anal.Appl.298(2004)

10.S. Kamimura, W. Takahashi, Approximating solutions of maximal monotone operators in Hilbert spaces, J. Approx. Theory 106 (2000) pp:226-240.

11.Z. H. Mabeed, Strongly Convergence Theorems of Ishikawa Iteration Process With Errors in Banach Space, Journal of Qadisiyah Computer Science and Mathematics, 3(2011)pp:1-8.

$12 . \mathrm{Z}$.H. Maibed, Strong Convergence of Iteration Processes for Infinite Family of General Extended Mappings, IOP Conf. Series: Journal of Physics: Conf. Series 1003

(2018) 012042 doi :10.1088/17426596/1003/1/012042.

13.S.S.Abed and Z.H .Maibed, Convergence Theorems for maximal monotone operator by family of nonspreading mappings(IJSR)PP.23197064.

14.Z.H .Maibed, Z.H .Maibed, J. of university of Anbar for pure science : Vol.7:NO.2( 2013 ).

15. S .Lemoto and W. Takahashi, Approximating Common Fixed Points Of Nonexpansive Mapping and Nonspreading Mappings In Hilbert Space, Nonlinear Analysis,71,(2009)pp:2082_2089. 
16. K . Aoyama and F . kohsaka and W. Takahashi., Proximal Point Methods for Monotone operator in Banach space, Taiwanese Journal of Math ,Vol.15 ,No.1, pp (259-281)2011.

17.K. K. Tan and H. K . XU , Approximating Fixed Points Of Nonexpansive Mappings By The Ishikawa Iteration process ,J .Math .Anal.Appl.178(1993),pp:301308.

18.S. Reich, "A Weak Convergence Theorems For The Alternative Method With Bregman Distance In Kartastos, AG (ed) theory and applications of nonlinear operators of Accretive and monotone type", Dekker New - York (1996)pp: 313 - 318.
19.D .A .Ruiz ,G. L. Acedo and V.M.Marquez, "Firmly nonexpansive mappings",J. Nonlinear analysis ,vol15(2014)1.

20.W. Takahashi and M. Toyoda, "Weak Convergence Theorem For Non-expansive Mappings and Monotone Mappings", J. optim theory Appl. 118 (2003) pp:417-428.

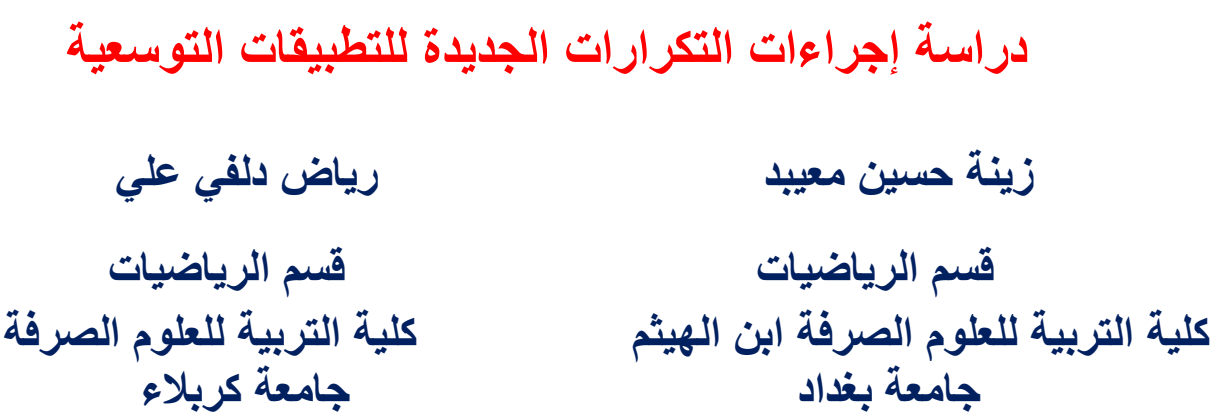

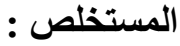

في هذا البحث سنقدم عمليات تكر ارية جديدة لانواع مختلفة من التطبيقات وسنقدم مفهوم التطبيقات التوسعية و التي تكون مستقلة عن التطبيقات الغير توسيعية .ايضا سندرس التقارب لهذا النوع من التكر ارات الى نقطة صامدة مشتركة في فضاء هلبرت 